\title{
THROUGHPUT OPTIMIZATION IN MULTI-RADIO MULTI- CHANNEL WIRELESS NETWORKS USING NETWORK CODING
}

\section{Saleh Dadpour, Reza Ghazizadeh \& Mohammad Sadeghian Kerdabadi University of Birjand, Birjand, Iran}

sdadpour@birjand.ac.ir;rghazizade@birjand.ac.ir;mohammad.sadeghian@ birjand.ac.ir

\begin{abstract}
One major problem of wireless mesh networks is low throughput and on the other hand, network coding (NC) is a reliable solution to alleviate this problem. In this paper, we evaluate the throughput gain of various intersession wireless NC schemes, including signal level (analog) NC and packet level NC, which may include non-duplex flows, over the traditional non-NC schemes in multi-radio, multi-channel and multi-hop networks. We also propose a routing approach in order to increase NC opportunities and evaluate its performance in wireless ad-hoc networks in terms of network throughput.
\end{abstract}

Keywords: ad-hoc network, throughput gain, network coding, routing, multiradio multi-channel.

\section{INTRODUCTION}

In wireless, mesh networks suffer from low throughput. Much effort has been put in to overcome this problem. On the other hand, network coding (NC) is known to be a promising technique to increase network throughput. Throuhput the use of network coding, intermediate nodes may combine packets together and forward them to the next hops instead of just forwarding them. By doing so, the information content of the packets is increased which results in throughput improvement of the network. Consider the scenario in Figure 1 in which Alice and Bob want to exchange packets. Since they are not in communication range of each other, a relay is needed to convey their packets. 


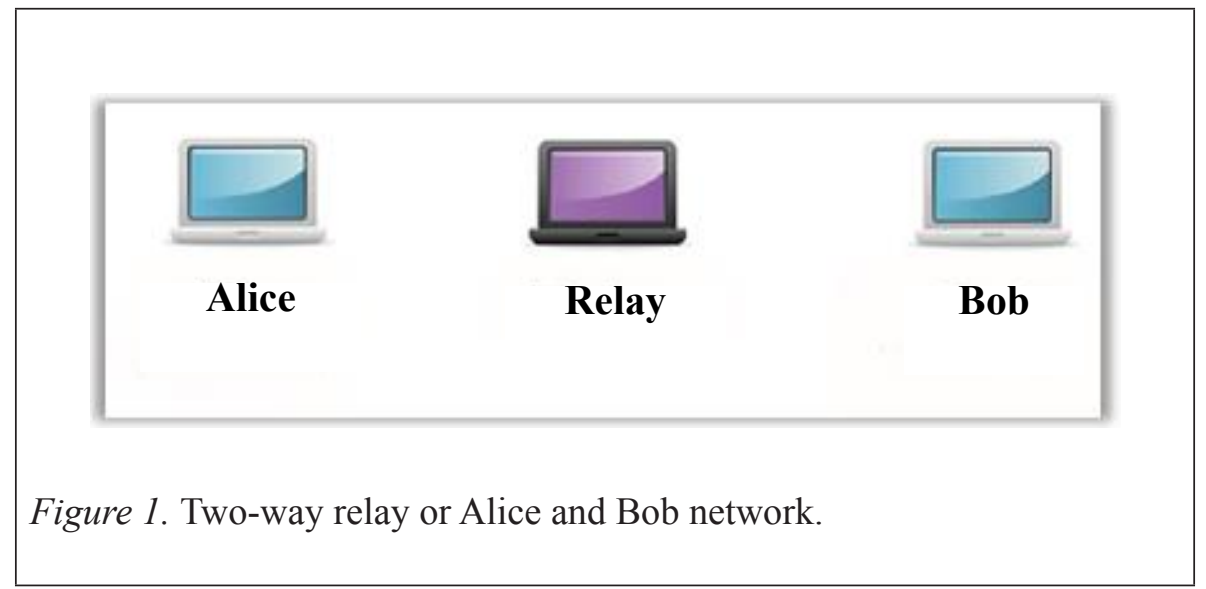

Without using network coding, four time slots are needed to exchange packets. The first two slots for sending Alice's packet to Bob and the remaining two slots for sending Bob's packet to Allice. In contrast, this can be done in three time slots by the use of conventional network coding (CNC) $(\mathrm{Wu}$, Chou, \& Kung, 2005; Fragouli, Boudec, \& Widmer , 2006). In the first two time slots, Alice and Bob send their packets to relay. Relay would combine the received packets, simply by an XOR operation, and then broadcast the result in the third time slot. Alice can obtain Bob's packet by performing an XOR operation between her own packet and the received packet from relay. In a similar way, Bob could decode Alice's packet. Here, the broadcast nature of the wireless medium is a good factor for wireless network coding. The possibility of mixing packets naturally in wireless medium is another property which could help improve the wireless network coding performance. This idea leads to analog network coding (ANC) (Katti et al., 2007; Shengli, 2006). Through the use of analog network coding in the two-way relay of Figure 1, Alice and Bob can exchange their packets in just two time slots. In the first slot, Alice and Bob send their packets to relay simultaneously. Thus, the signals will interfere in the relay. Then, in the second slot the relay would broadcast the naturally mixed signal. Through the use of network level information, the receiver tries to cancel the interference and get its desired signal. Both the NC scenarios mentioned above, were designed for the bidirectional relay of Figure 1. However, few applications send data in both directions in practice. Wireless network coding can be extended to the more generalized " $X$ " and " $Y$ " topologies of Figure 2 through the use of “opportunistic listening" (Katti et al., 2008). 


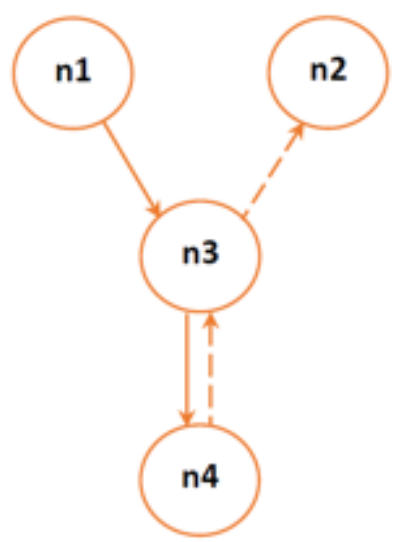

(b)

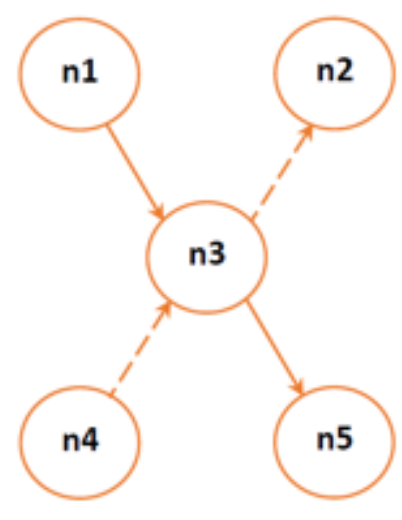

(a)

Figure 2. (a) X topology, (b) Y topology.

In Figure 2 (a), two sessions should be held. One from $n_{1}$ to $n_{5}$ with the path specified by solid lines, and the other from $n_{4}$ to $n_{2}$ with the path specified by dash lines. In order to perform network coding in this topology, in the first time slot $n_{1}$ sends its packet to $n_{3}$, and meanwhile, $n_{2}$ which is in the communication range of $n_{1}$ overhears it. In the second slot, $n_{4}$ sends its packet to $\mathrm{n}_{3}$ and similarly $\mathrm{n}_{5}$ in the communication range of $\mathrm{n}_{4}$ would also overhear it. In this time, $\mathrm{n}_{3}$ would combine (XOR) these two packets and broadcast the result in the third time slot. Now, $\mathrm{n}_{5}$ could get its desired packet simply by performing an XOR operation between the overheard packet sent by $\mathrm{n}_{4}$ and its received packet in the third time slot. In a similar way, $\mathrm{n}_{2}$ could also obtain its desired packet. Obviously, the NC technique applied in this topology is analogous to the conventional $\mathrm{NC}$ in the two-way Alice and Bob network and the combination of these two yields the Y-topology shown in Figure 2 (b). Because of this analogy, the NC technique used in both topologies of Figure 2 (a) and (b) can also be categorized in the conventional NC. However, for the ease of reference, we refer to the $\mathrm{X}$ and $\mathrm{Y}$ topologies of Figure 2 as "cross topology" in general.

In this paper, we expand the network throughput model of (Su et al., 2009) to encompass the cross topology scenarios and a modification in the dijkstra routing protocol is proposed to improve the performance of network coding in wireless mesh networks. 


\section{RELATED WORKS}

The notion of network coding was first introduced by Ahlswede et al. (2000) who showed that in wired networks, if the intermediate nodes are allowed to mix the received packets before forwarding, the multicast communication could achieve its capacity. Since then, many efforts have been done to improve network throughput both in wired and wireless networks through the use of network coding. Li et al., (2004); and Nazer et al. 2011) investigated how network coding can lead to throughput improvement in wireless networks. Yang et al. (2012), compared the existing network coding schemes such as $\mathrm{ANC}$ and $\mathrm{CNC}$ and introduced a method for the implementation of ANC in two-way relay networks. Besides, an in-depth analysis on the implementation issues of wireless $\mathrm{NC}$ considering the impact of resource constraints on the performance of $\mathrm{NC}$ in the content distribution application was presented in Lee et al, (2013). Katti et al. (2008) presented the COPE scheme, an idea of conventional network coding was which expanded to the cross topology. This scheme may increase the number of nodes involved in the network coding operation. A centrality-based network coding node selection (CNCNS) was proposed to minimize the number of $\mathrm{NC}$ nodes without compromising the achievable network throughput (Kim et al., (2014). Argyriou et al. $(2009,2010)$ proposed an optimized algorithm to implement the cross topology network coding introduced by (Katti et al. (2008) on the IEEE 802.11 networks. Xie et al. (2015) presented a virtual overhearing technique to enable $\mathrm{NC}$ when the traditional overhearing methods do not yield the required information. A lots of effort has been put into leveraging network coding in order to optimize the throughput of wireless ad-hoc networks. In Teav et al., (2012), a cooperative network coding protocol was proposed in a MIMO Y channel system in which three wireless nodes exchange information simultaneously via one relay in two time slots. Besides, a throughput optimization problem with a BER constraint was designed to maximize the throughput of the system. Joint scheduling and network coding in wireless multicast networks which were investigated by (Niati et al. (2012) included three successive steps: (a) scheduling, (b) optimization, and (c) coding. Furthermore, a linear optimization problem aimed to obtain schedule-specific link flows which are important in coding process was formulated. In Ayedi et al. (2015), the optimum distribution of relay energy in a C-ARQ system through the use of analog network coding was studied. The optimization was aimed to maximize the throughput subjected to an average relay energy constraint. Yan et al. (2012), developed a theoretical model to compute the achievable throughput of cooperative MCD in VANETs using SLNC. However, none of the previously mentioned works provided a general framework to investigate the network throughput gain in multi-hop, 
multi-channel, and multi-radio wireless ad hoc networks. Chieochan et al. (2013) proposed a general framework for throughput optimization in multiradio multi-channel wireless mesh networks using intra-session network coding. Unfortunately, for the most frequent scenarios in which only unicast sessions were presented, the benefits of intra-session network coding are vanished. Intersession network coding provides performance improvement even when only unicast sessions are presented (Wang et al., 2008). Choi et al. (2016) proposed a combined intra-session and intersession NC method in order to improve the network utility in multi-radio, multichannel, and multirate wireless network. Besides, a network throughput optimization scheme with a rate control, resource allocation and congestion control algorithm was proposed. But, the framework did not include ANC. Other framework were proposed by in Su et al.(2009) and Zhang et al.2009) in the case of intersession NC for wireless ad-hoc and wireless mesh networks respectively, in which the optimal throughput gain of the primitive wireless $\mathrm{NC}$ scenarios, i.e. $\mathrm{CNC}$ and $\mathrm{ANC}$, are characterized by the use of linear programming. However, the studied NC scenarios were confined to combinations of the bi-directional two-way relays of Figure 1. Executing NC in non-duplex flows such as those shown in Figure 2 were not considered in the framework. We extended the framework in Su et al.(2009) to include such cases too.

\section{NETWORK MODEL}

The network model is characterized by a set of nodes and the links corresponding to the pair of nodes are in communication or interference range of each other. Let's denote the set of nodes, communication links and interference links by $\mathrm{V}, \mathrm{E}$ and I, respectively. Note that $\mathrm{E}$ is the set of links which can transfer the data, but I is a set of links which were able to sense the data, but cannot decode them. The directed link from node $\mathrm{s}$ to node $\mathrm{t}$ is denoted by $e=(s, t)$, and $\bar{e}=(t, s)$, is the reverse link of e. Some sessions should be held between the pairs of source and the destination nodes in the network. The set of all sessions is denoted by A. A session $e_{i} \in \mathrm{A}$ is characterized by a triplet $\left\{s\left(a_{i}\right), d\left(a_{i}\right), \theta\left(a_{i}\right)\right\}$, where $s\left(a_{i}\right), d\left(a_{i}\right)$ and $\theta\left(a_{i}\right)$ are the source node, the destination node and the requested throughput of session $a_{i}$ respectively. Each node in the network can be a source or destination. The packets of $a$ session can be routed in multi hops if the source and the destination nodes are not in communication range of each other. There may be multiple paths for a session in multi-path routing scenarios. With the decline in IEEE 802.11 equipment costs, a node may have multiple radios to support simultaneous transmissions over orthogonal frequency channels. The total number of orthogonal frequency channels in the wireless ad-hoc network is supposed to be $M$. 


\section{NC links combinations}

NC links combination is defined as a set of communication links which can perform a network coding operation together. The existence of NC links combinations in a network depends on the routes of various sessions. In particular, if there exists three nodes which could form a two-way relay subnetwork, both CNC and ANC scenarios could be implemented.

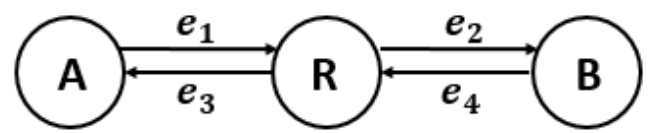

Figure 3. Two-way relay subnetwork which is an opportunity to perform $\mathrm{ANC}$ and $\mathrm{CNC}$.

In Figure 3, we have,

$\left\{e_{1}, e_{2}\right\} \in a_{1}$

$\left\{e_{4}, e_{3}\right\} \in a_{2}$

$a_{1} \neq a_{2}$

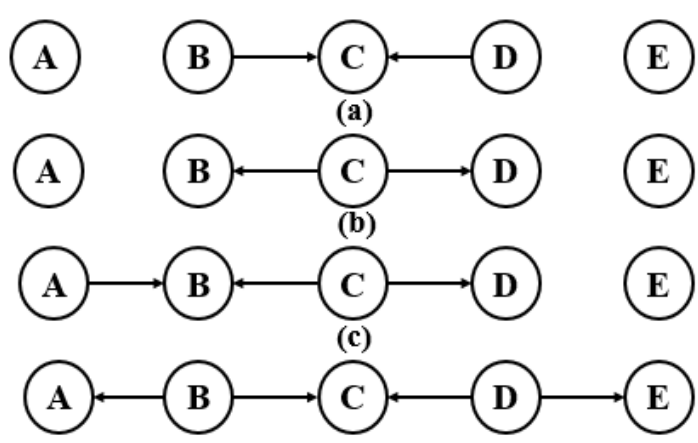

(d)

Figure 4. Various kinds of NC links combinations: (a) Incoming NC links combinations (b) outgoing NC links combinations, (c) 4-node NC links combinations and (d) 5-node NC links combinations.

In such cases, $\left\{e_{1}, e_{4}\right\}$ is defined as incoming $\mathrm{NC}$ links combination and $\left\{e_{2}, e_{3}\right\}$ is defined as outgoing $\mathrm{NC}$ links combination. Incoming and outgoing $\mathrm{NC}$ links combinations may be further combined to a more powerful NC links 
combination. All unicast links involved in an NC links combination should be active at the same time. Thus, more links involved in an NC links combination leads to more network efficiency. However, due to synchronization problems, $\mathrm{NC}$ links combinations which contain more than one incoming NC links are not considered ( $\mathrm{Su}$ et al., 2009). As a result, the set of all possible kinds of NC links combinations are shown in Figure 4.

\section{PROBLEM FORMULATION}

In this part, a brief description on the throughput optimization program is presented, while more details can be found in Su et al. (2009). Moreover, the complete set of the program is presented in "APPENDIX A". Let $F(a)$ be the flow weight of session $a$. Larger $F(a)$ is equivalent to a larger throughput requirement from session $a$. The objective of the problem is to maximize $\lambda$ such that at least $\lambda F(a)$ amount of throughput is supplied for each session $a$, with $a \in A$. The optimization's constraints are described in below.

Link flow constraint: Two types of traffic are considered for each communication link $e \in E$ : Unicast traffic and NC traffic. The flow of unicast traffic on link $e \in E$ and the flow of NC traffic on link e associated with the NC links combinations $L \in L(e)$ are denoted by $f_{m}^{U}(e)$ and $f_{m}^{N C}(L)$ respectively on channel m. $L(e)$ is the set of NC links combinations associated with link $e$. Thus, the total amount of flow on link $e \in E$ is the sum of all unicast flow and NC flow over all channels. At the same time, the total amount of flow on link $e$ should be equal to the total flow of all paths which have routes through link $e$.

Flow conservation constraint: The flow conservation constraint is used to maintain the flow balance at each wireless node. In each wireless node which does not initiate or terminate $a$ session, the total amount of outgoing flow should be equal to that of incoming flow.

Transmission interference constraint: In wireless communication, when a unicast link or an $\mathrm{NC}$ links combination is active on channel $\mathrm{m}$, all its conflict links at the same channel should be muted. In other words, every unicast link or NC links combination should share the same medium. Normalized active time could be used to model this constraint, that is,

$$
\sum_{e^{\prime} \in E^{c}(e)} x_{m}^{U}\left(e^{\prime}\right)+\sum_{L \in L_{m}^{C}(e)} x_{m}^{N C}(L) \leq 1
$$


where $E^{c}(e)$ is the set of unicast links which interfere with the link $e, L_{m}^{C}(e)$ is the set of conflict NC links of link $e$ in channel $m . x_{m}^{U}(e)$ and $x_{m}^{U}(e)$ is the fraction of time in which unicast link $e$ and NC links combination $L$ are active over channel $m$, respectively, i.e.

$$
\begin{gathered}
x_{m}^{U}(e)=\left\{\begin{array}{cc}
\frac{f_{m}^{U}(e)}{c_{m}(e)}, & c_{m}(e)>0 \\
0, & \text { otherwise }
\end{array}\right. \\
x_{m}^{N C}(L)=\left\{\begin{array}{cc}
\frac{f_{m}^{N C}(L)}{\min _{e \in L}\left\{c_{m}(e)\right\}}, & \min _{e \in L}\left\{c_{m}(e)\right\}>0 \\
0, & \text { otherwise }
\end{array}\right.
\end{gathered}
$$

Thus, the first and second terms of Eq. (1) denote the normalized active time for unicast and NC links respectively.

Link capacity constraint: This constraint is to insure that the link flow for each unicast link or NC links combination does not go above its upper bound. Node-radio constraint: The number of channels a node can simultaneously access is equal to the number of radios that the node is equipped with. The number of active unicast links and active NC-links combinations of each node should not go beyond the number of radios equipped by the node at the same time.

After specifying the above constraints, a linear program (LP1) could be finalized to find the flows which maximize $\lambda$ the under above constraints. This LP1 represents a general form for throughput optimization of the non-NC, conventional NC and analog NC schemes. In particular, if we let $L(e)=\varnothing, \forall$ $e \in E$, then LP1 will represent throughput optimization problems for the non-NC scenario. If we consider only the outgoing NC-links combinations as the allowed NC-links set, then LP1 will become the conventional NC scenario optimization problem. If both the incoming and the outgoing NClinks combinations are allowed, LP1 will become the analog NC scenario optimization problem. Finally, if all types of NC-links combinations introduced in Figure 4 are allowed, we will have the NC5 scenario optimization program.

Traffic flow optimization: The LP1 program described in the previous section yields the optimal flows which maximizes the overall throughput. Let the optimum solution of LP1 be $\lambda^{*}$. Although the solution of LP1 obtains the unique and optimum $\lambda$, the unicast and NC-link flows which lead to the optimum $\lambda$ are not unique. Hence, the obtained link flows from LP1 may not 
be optimum from the interference aspect. Therefore, it will be practical that for every link, among all the flows which yield $\lambda^{*}$, find the optimum one such that the total interference is minimized. For this purpose, the second linear program denoted by LP2 could be defined with the objective of minimizing the total interference between any two links. The objective of LP2 could be set as follows

$$
\text { minimize } \sum_{m \in M}\left[\sum_{e \in E} x_{m}^{U}(e)+\sum_{L \in L} x_{m}^{N C}(L)\right]
$$

Where $x_{m}^{U}(e)$ and $x_{m}^{N C}(L)$ are normalized active time for unicast and NC links respectively. The constraints of LP2 are similar to those of LP1. The only difference is that the optimum throughput, $\lambda^{*}$ is known here.

\section{Proposed routing strategies}

A given session can have either a single path or multi-paths. Both are considered in this article. Intuitively, multi-path routing schemes provide more network coding opportunities compared to single-path schemes. However, multi-path routing may not be applicable in some cases due to high routing maintenance overheads. Thus, the conventional shortest single-path routing may be modified to provide more coding opportunities. For this purpose, one solution is to select the path which provides the maximum flow in multi-path routing for each session (Su et al., 2009). This can be done in two steps. First, LP1 is solved with multi-path routing. Then, in the second step, the path which provides the maximum flow for each session is selected, i.e.

$$
p=\arg _{p \in P_{a}} \max F_{a}(p)
$$

In Eq. (5), $P_{a}$ is the set of edge-disjoint paths of session $a$. These selected paths are inputs to LP1 for throughput optimization. Another solution is to encourage the routing protocols to deliberately increase the probability of interference. Traditionally, interference is not welcome in wireless communications. However, in the case of deploying NC, it can be helpful in providing more $\mathrm{NC}$ opportunities and consequently improving the network throughput (Katti et al., 2007). To do this, our proposed strategy was to offer a discount in the routing cost of the reverse links of currently active paths in the network. Such links have a great potential to provide NC opportunities in the network. In this way, if some new sessions are introduced to the network, the routing protocol will prefer to route them via the links subjected to discount. Let us illustrate 
this with an example. Figure 5 shows a grid network in which 16 nodes are distributed in a $4 \times 4$ units rectangular area. The destination between two adjacent nodes is 1 unit. Communication range and interference range are 1 and 1.4 units respectively. Without loss of generality, we assume that all the links have the same capacity (and the same routing cost). Thus, they can be normalized to one. One session from node A to node B is currently active in the network which is routed through the path specified by blue arrows. Now, assume, after a while, another session from node B to node A is initiated in the network. If no modification in the links' routing costs occurs, the routing path of the new session can be the one specified by the dotted red arrows. In this case, no NC opportunity exists in the network. But, if a discount is offered to all of the reverse links of session 1's routing path ( $\overline{e_{1}}$ to $\overline{e_{6}}$ links), for instance, their cost decreases from 1 to 0.8 ; the new path will be the solid red arrows in Figure 5. In this case, there are many opportunities to implement NC which are listed below.

Incoming NC links: $\left(\left\{e_{1}, \overline{e_{2}}\right\},\left\{e_{2}, \overline{e_{3}}\right\},\left\{e_{3}, \overline{e_{4}}\right\},\left\{e_{4}, \overline{e_{5}}\right\},\left\{e_{5}, \overline{e_{6}}\right\}\right)$

Outgoing NC links: $\left(\left\{\overline{e_{1}}, e_{2}\right\},\left\{\overline{e_{2}}, e_{3}\right\},\left\{\overline{e_{3}}, e_{4}\right\},\left\{\overline{e_{4}}, e_{5}\right\},\left\{\overline{e_{5}}, e_{6}\right\}\right)$

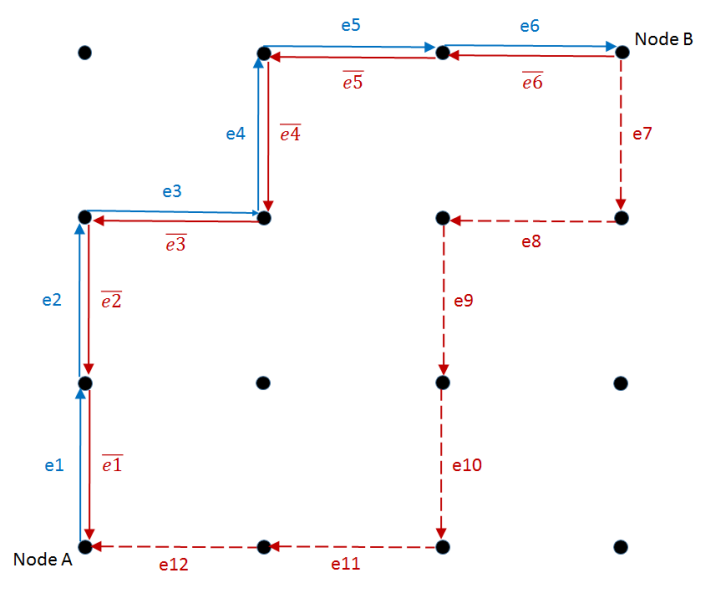

Figure 5. The effect of proposed routing price modification on the selected path.

After finding the new path, it is not necessary anymore to keep the offered discount for the links. Thus all the costs return to their default value. In essence, our idea is that whenever a routing process for a session is accomplished, a discount will be offered to its reverse links. Then, if any new upcoming session 
selects a discounted link, our goal for discounting is met and discounting is not necessary anymore. Thus, the link's price should return to its previous value. Our proposed routing method is faster than the one introduced in Su et al. (2009). Because the algorithm in Su et al. (2009) needs to perform multipath routing and solve the LP1 in this mode in order to decide the appropriate routes in the single-path mode, it has to perform LP1 again to optimize the throughput. But, our algorithm needs to execute the LP1 once and perform the routing in the single-path mode. Furthermore, it is not confined to single- path routing.

\section{PERFORMANCE EVALUATIONS}

\section{Evaluation setups}

In this section, we evaluate various wireless $\mathrm{NC}$ scenarios when multiple unicast sessions are presented in the network. Our simulations were conducted

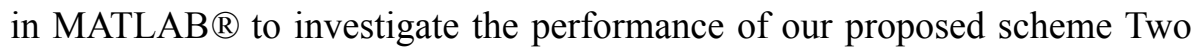
common scenarios for network topology are shown in Figure 6; random network and grid network (Su et al., 2009).

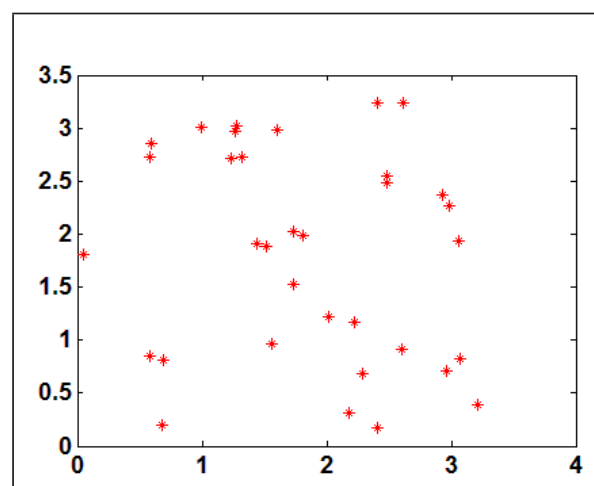

(a)

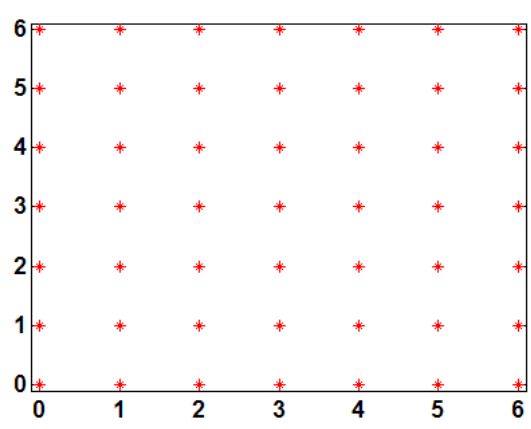

(b)

Figure 6. The scenarios for network topology (a) Random network (b) Grid network.

In the random network, it was assumed that 34 nodes were distributed randomly in a $3.4 \times 3.4$ units rectangular area. In the grid network, 49 nodes were distributed in a $7 \times 7$ units rectangular area. The wireless channel was assumed to be free a space model. For simplicity, all wireless channels in the network were supposed to have equal capacities. Besides, we assumed 
that all sessions had equal flow weights, i.e. $\mathrm{k}(a)=\mathrm{k}(b)=1, \forall \mathrm{a} \neq \mathrm{b} \in \mathrm{A}$. Finally, all wireless nodes had the same number of radios and all radios had equal number of channels. The destination between two adjacent nodes was 1 unit. Communication range and interference range were 1 and 1.4 units respectively. Based on these ranges, each node identified its neighbors in the network. Then, the sessions were specified. For each session, two distinct nodes were selected randomly as source and destination. After specifying the sessions, routing process was executed. We studied the simple singlepath and multi-path routing by means of the dijkstra method in two different circumstances. In the first one, no routing cost modification was considered. Then, in the second one, the price modification described in the previous section was executed. Besides, the optimized single-path routing proposed in $\mathrm{Su}$ et al. (2009) was studied. After the routing process, the active links which can form NC links combinations were identified. We investigated the non-NC, conventional, conventional-plus, analog and advanced analog NC scenarios. The analog network coding scenario only deployed the incoming and outgoing NC links combinations shown in Figure 4 (a) and (b) referred to as analog $\mathrm{NC}$ or NC3. Besides, the one which was allowed to use all of the four NC-links combinations shown in Figure 4 was referred to as advanced analog NC or NC5. Through the use of NC3 and NC5 scenarios, a significant boost in throughput gain of the network was expected. However, precise synchronization techniques and accurate channel state information was required to implement the ANC scenarios. As a result, the implementation of these methods was not possible in some circumstances. On the other hand, in a wireless network, it is expected that many flows intersect on a common node, thus many opportunities for implementation of cross topology NC may arise. Besides, no complicated conditions such as those of the ANC techniques is required for the implementation of cross topology NC. As a result, neglecting the practical difficulties of implementation, but obtaining the promised throughput gain improvement of ANC by means of cross topology NC, was of great interest to us. To evaluate the efficiency of this idea, we defined a subcategory of the conventional $\mathrm{NC}$ scheme which was allowed to use the cross topology $\mathrm{NC}$ technique shown in Figure 2 in addition to the incoming NClinks of Figure 4 (a). We refer to this sub-category as Conventional-plus NC. For the ease of reference, we summarized the above mentioned discussions in Figure 6.

By solving LP1 which can be found in Appendix A, we obtained the throughput for each of the various $\mathrm{NC}$ scenarios mentioned in Figure 7 and in various unicast session numbers. Then, we calculated the network throughput gain with respect to the non-NC case. 


\begin{tabular}{|l|c|c|c|c|c|}
\hline & $\begin{array}{c}\text { Incoming } \\
\text { NC links }\end{array}$ & $\begin{array}{c}\text { Outgoing } \\
\text { NC links }\end{array}$ & $\begin{array}{c}\text { 4-node } \\
\text { NC links }\end{array}$ & $\begin{array}{c}\text { 5-node } \\
\text { NC links }\end{array}$ & $\begin{array}{c}\text { Cross } \\
\text { NC links }\end{array}$ \\
\hline Non-NC & & & & & \\
\hline $\begin{array}{l}\text { Conventional } \\
\text { NC }\end{array}$ & & & & & \\
\hline NC3 & & & & & \\
\hline NC5 & & & & & \\
\hline $\begin{array}{l}\text { Conventional } \\
\text {-plus NC }\end{array}$ & & & & & \\
\hline
\end{tabular}

Figure 7. Various scenarios of NC-links combinations.

\section{The throughput gain of wireless $N C$ schemes}

In this section, we investigate the network throughput gain in case of using each of the NC scenarios shown in Figure 7, with respect to the non-NC scenario. First, we assumed each node had only one radio and each radio had just one channel. Figure 8 shows the wireless NC performance in the random network. The results in Figure 8 show that the conventional-plus NC significantly outperforms in comparison with any other NC scheme. This is not surprising because there will be more NC opportunities if cross NC links are allowed.

More NC opportunities result in more network throughput. Furthermore, it can be seen in Figure 7 that in light traffic in which the number of sessions are not too much, the performance of NC3 and NC5 are the same and even in higher levels of traffic, there is no significant difference between these two NC schemes. That is because there are not many opportunities of finding $L_{4}$ or $L_{5} \mathrm{NC}$ links in the random network. However, the performance gap between conventional $\mathrm{NC}$ and $\mathrm{NC} 3$ is relatively high which means there are many opportunities of incoming NC links compared to outgoing NC links in the network. Figure 8 shows the effect of deploying various NC scenarios in the network throughput gain of a grid network. 

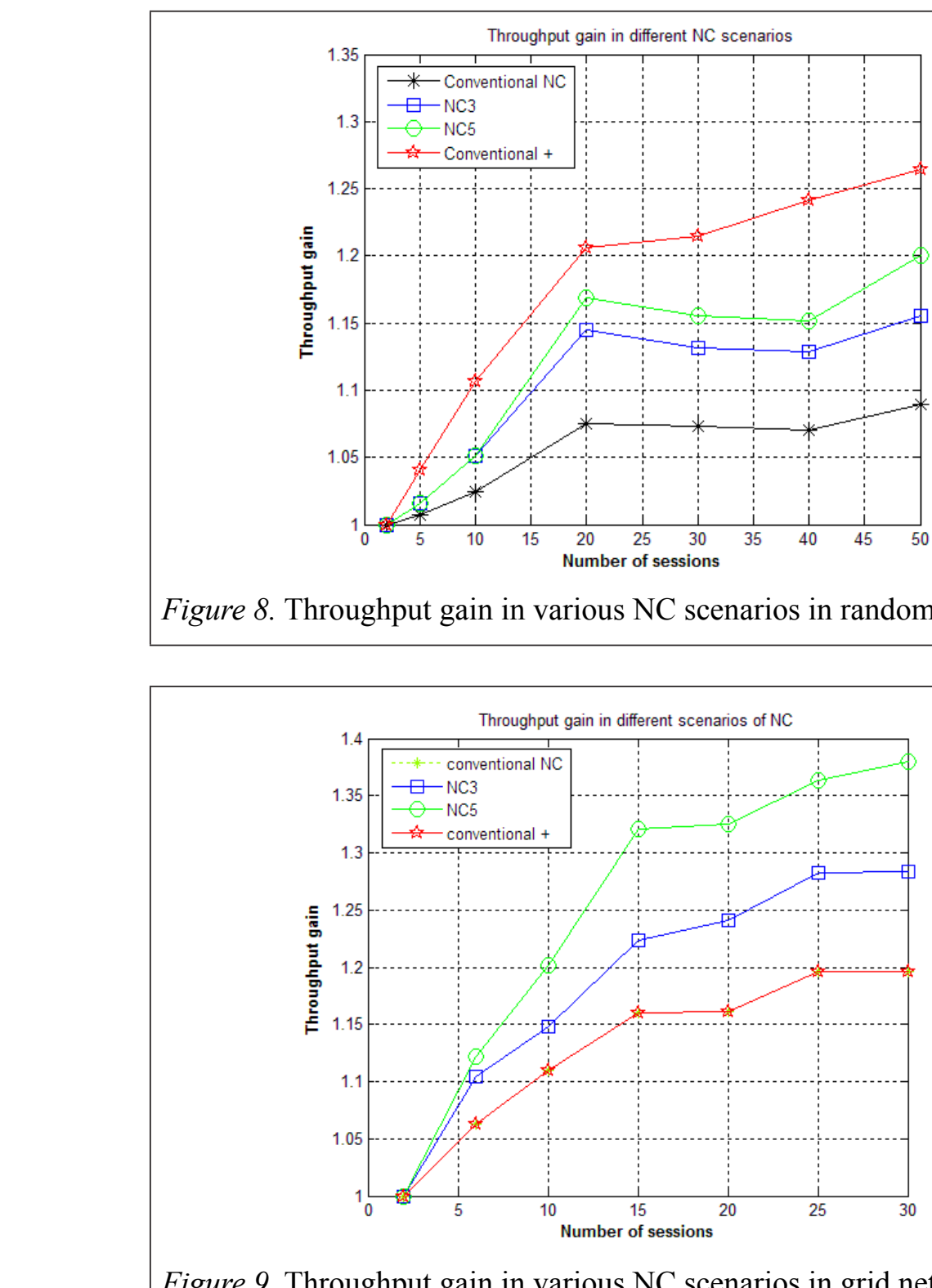

Figure 8. Throughput gain in various NC scenarios in random network.

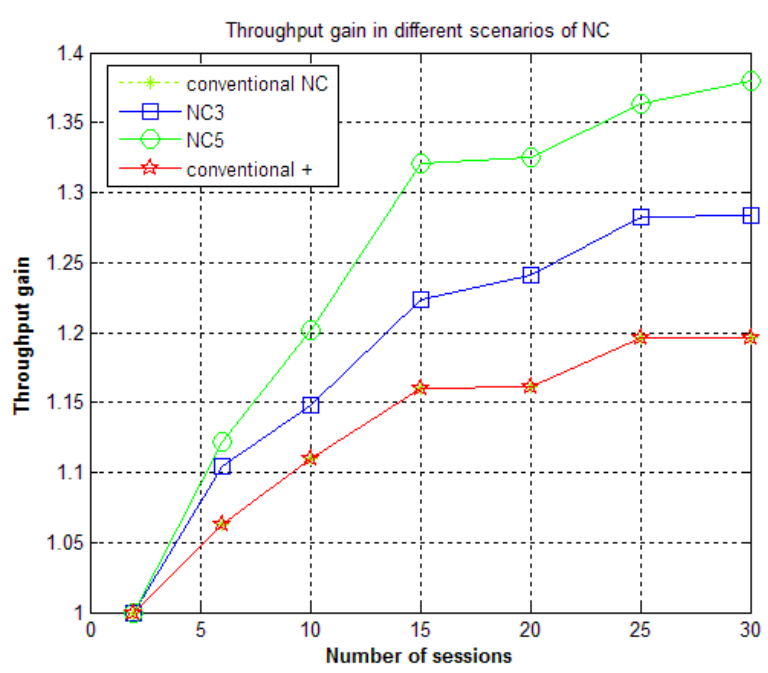

Figure 9. Throughput gain in various NC scenarios in grid network.

It can be seen that the performance of the conventional and conventional plus $\mathrm{NC}$ schemes are the same because in this case, there is no chance to find cross NC links. However, contrary to the random network case, there is a relatively huge gap between the performances of the NC3 and NC5 schemes, especially in high traffic loads. That is because there are many opportunities of finding $L_{4}$ or $L_{5} \mathrm{NC}$ links in a grid network. 


\section{Performance impact of routing strategies}

In this section, we evaluate the effect of some different scenarios of routing such as simple dijkstra single-path and multi-path routing, optimized singlepath routing and our proposed routing scenario in both the single and multipath routing schemes .Figure 10 and Figure 11 show the effect of various routing scenarios in throughput gain of conventional and analog $\mathrm{NC}$ schemes respectively, when deployed in the random network.

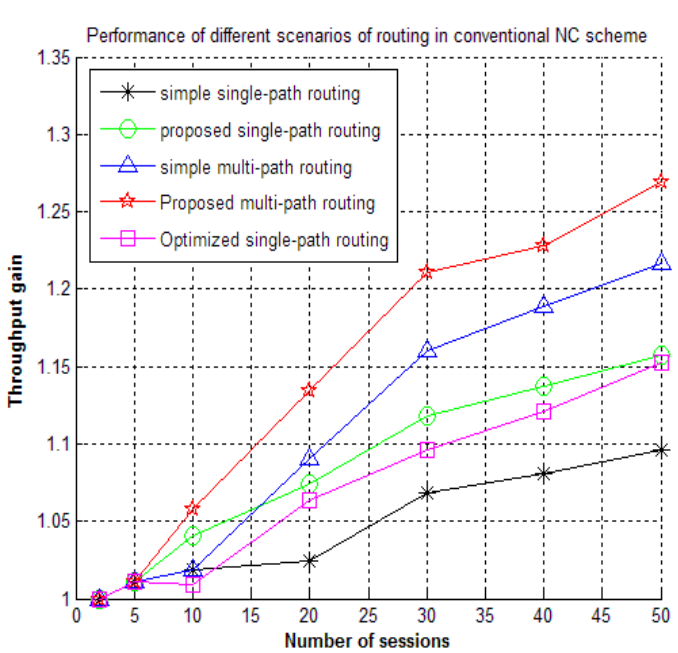

(a)

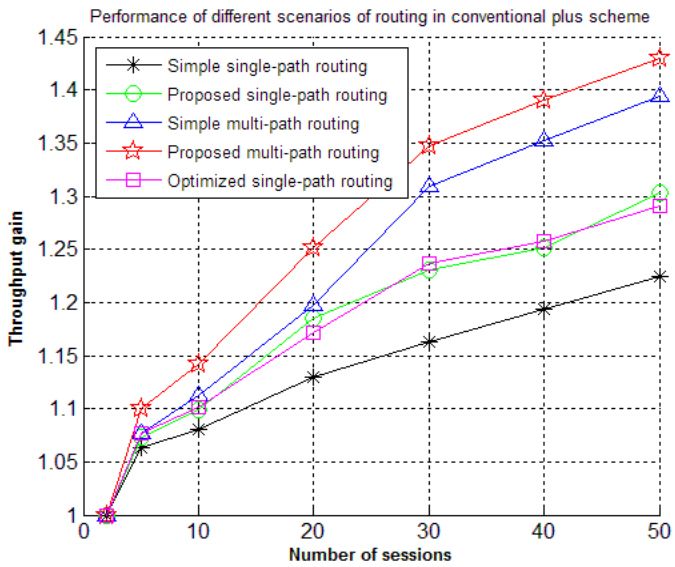

(b)

Figure 10. Throughput gain in various routing scenarios in random network and in the case of using conventional NC schemes (a) conventional NC and (b) conventional-plus NC. 


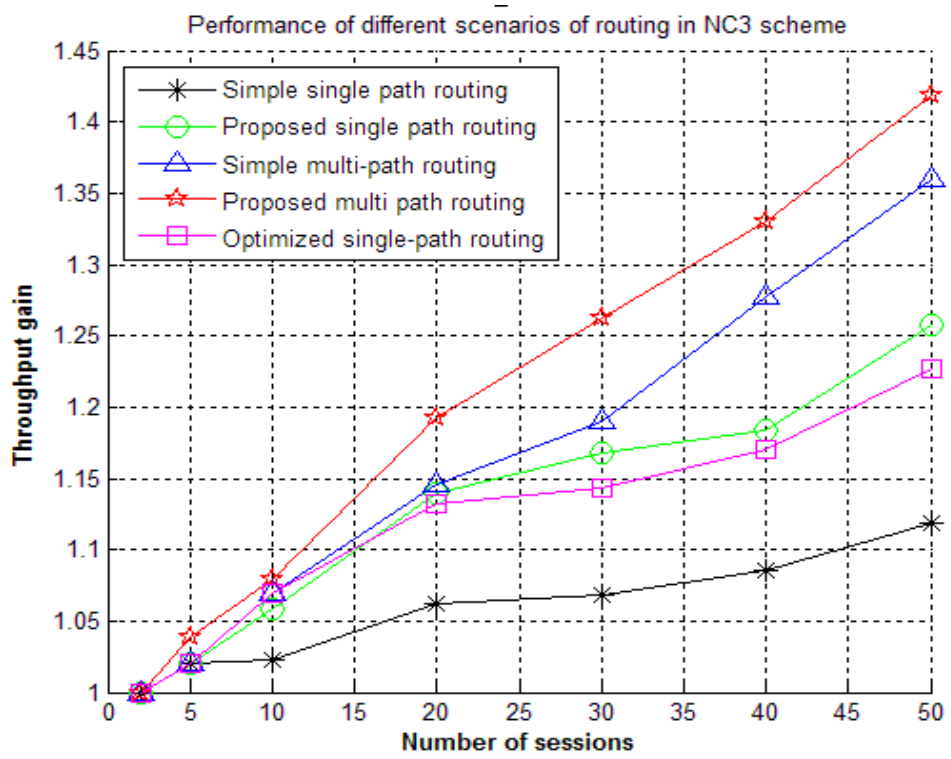

(a)

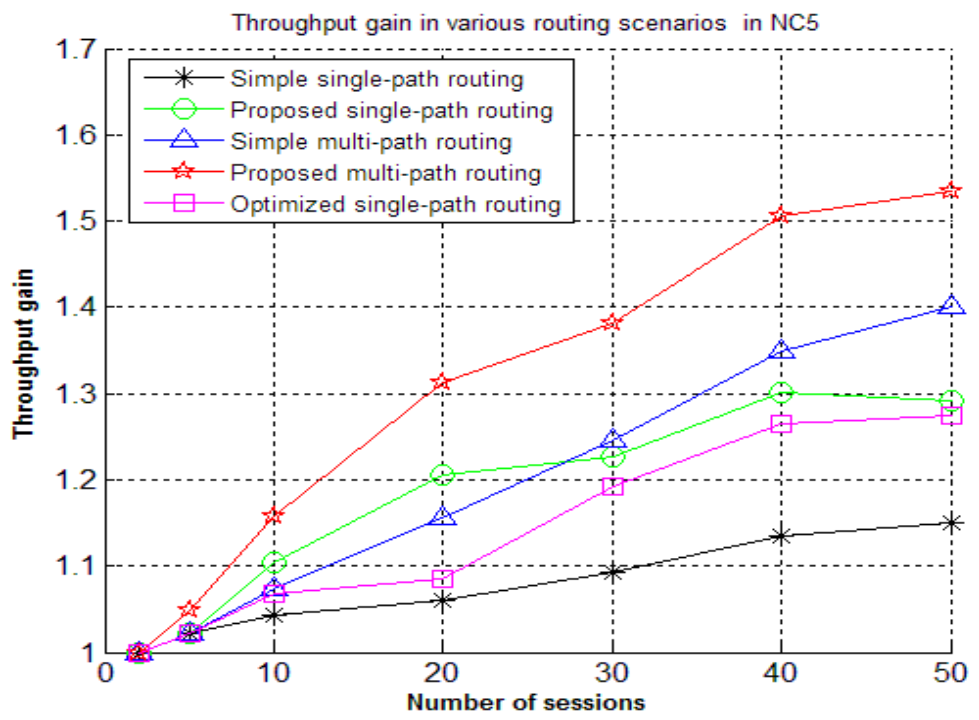

(b)

Figure 11. Throughput gain in various routing scenarios in random network and in the case of using analog NC schemes (a) NC3 scheme and (b) NC5 scheme. 
In all the NC scenarios, there was no significant difference in the throughput gain of our proposed single-path routing and optimized single-path routing in heavy traffic. However, in Figure 10, the performance gap in the conventional $\mathrm{NC}$ is larger compared to the conventional-plus scenario. It is because in the conventional NC, just two-way Alice and Bob NC opportunities were considered and our proposed routing was primarily aimed to increase this kind of NC opportunities. Increasing cross topology NC opportunities is only a side effect. On the other hand, in conventional-plus NC, the dominant factor for the improvement of network throughput gain is cross topology NC opportunities. Because in a real wireless network, there are usually much more opportunities to perform cross topology NC. Although there is not a considerable difference in throughput gain of these two NC scenarios, our proposed routing was faster and easier to implement. Furthermore, it was not confined to singlepath routing. Usually, there are not many opportunities to perform NC in light network traffic unless we increase the NC opportunities artificially. This was what our proposed routing method did. Thus, in some cases, such as in Figure 10 (a) and Figure 11 (b), the performance of the proposed single-path routing in light traffic is even better than that of the simple multi-path routing. However, as we move toward the heavy traffic region, the NC opportunities in multipath scenarios ascend with a high slope such that finally it overtakes that of the single-path schemes. Our proposed routing was not limited to single-path routing. Based on the results in Figure 10 and Figure 11 our proposed routing can improve the performance of the multi-path routing scheme considerably.

\section{Performance impact of multi-radio multi-channel}

In this section, we investigate how multi-radio multi-channel affects the performance of network coding in a wireless network. Figure 12 shows the effect of multi-channel on the throughput gain of the conventional-plus NC scenario when each node is equipped with only one radio in two different routing methods. According to the results in Figure 12 and Figure 13, when we gradually increase the number of channels, at first, the throughput gain of the random network in a fixed number of sessions increases almost linearly. But suddenly, the throughput gain slope experiences a sharp drop and then, the further increase in the number of channels has nothing to do with the throughput gain. In other words, throughput gain has a saturation capacity. This result is important because in most cases, we have a limited resource of bandwidth. Thus, splitting the available bandwidth to more frequency channels results in shrinking each frequency channel bandwidth which affects the transmission rate. For this reason, there should be a trade-off between network throughput and available channel BW. 


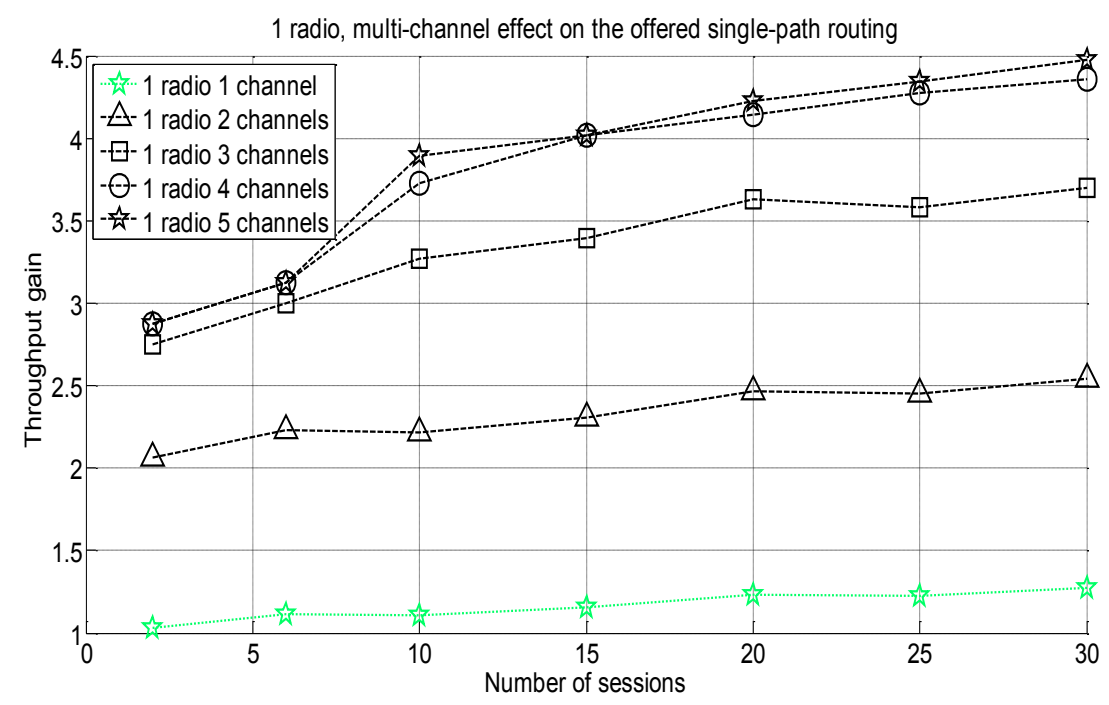

(a)

1 radio, multi channel effect on the offered multi path routing

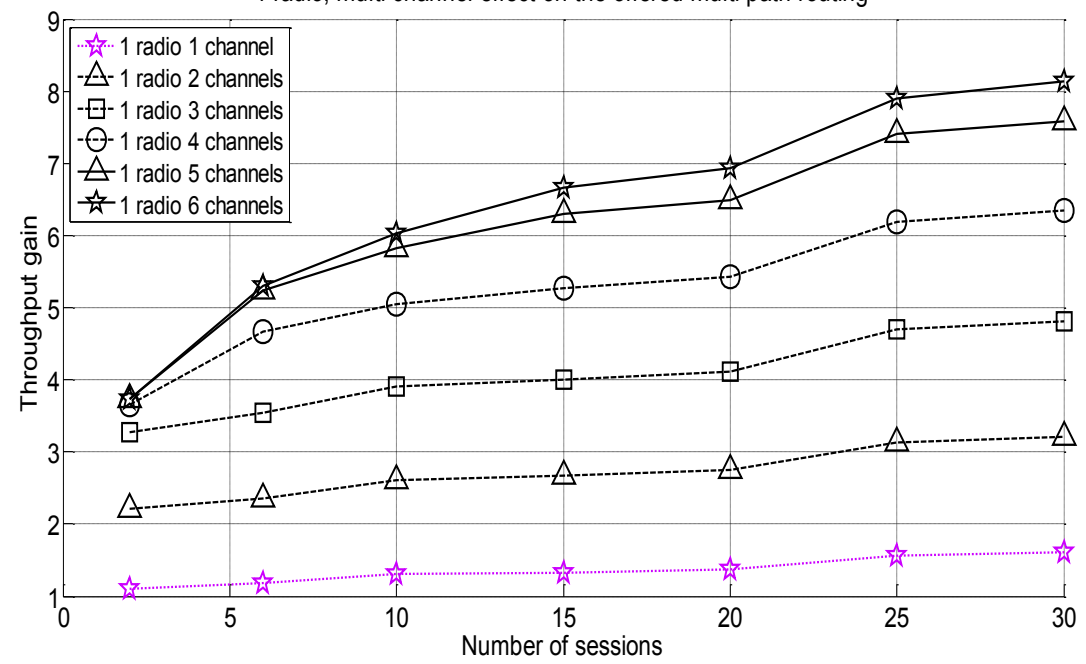

(b)

Figure 12. Effect of multi-channel on the throughput gain in conventional plus and random network when each node is equipped with 1 radio in 2 different routing scenarios (a) Proposed single-path routing, (b) Proposed multi-path routing. 


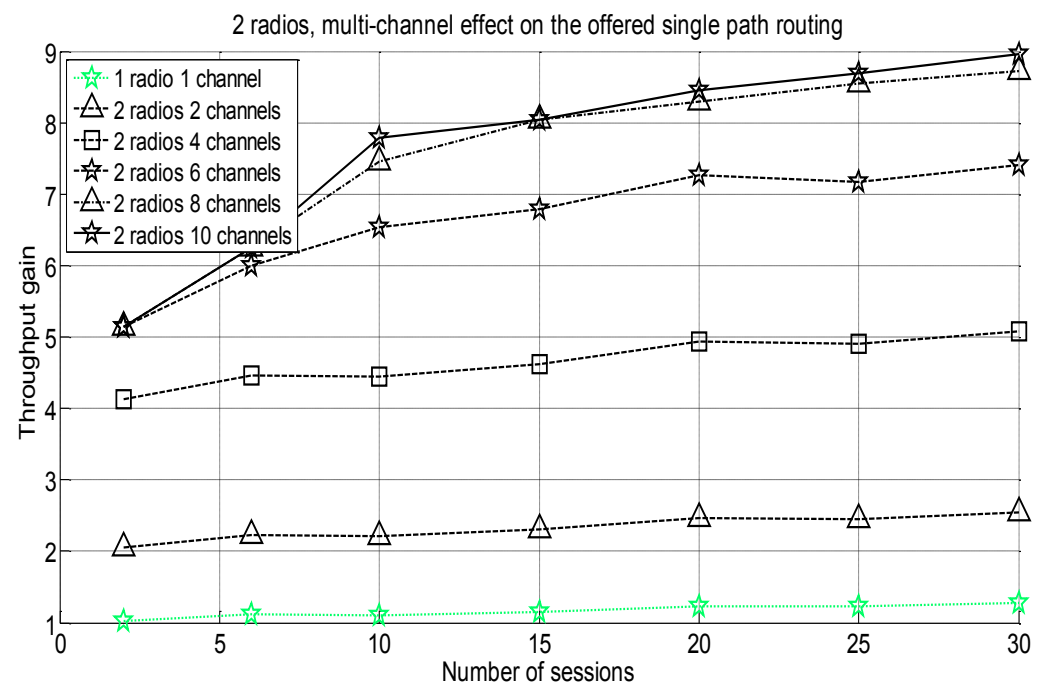

(a)

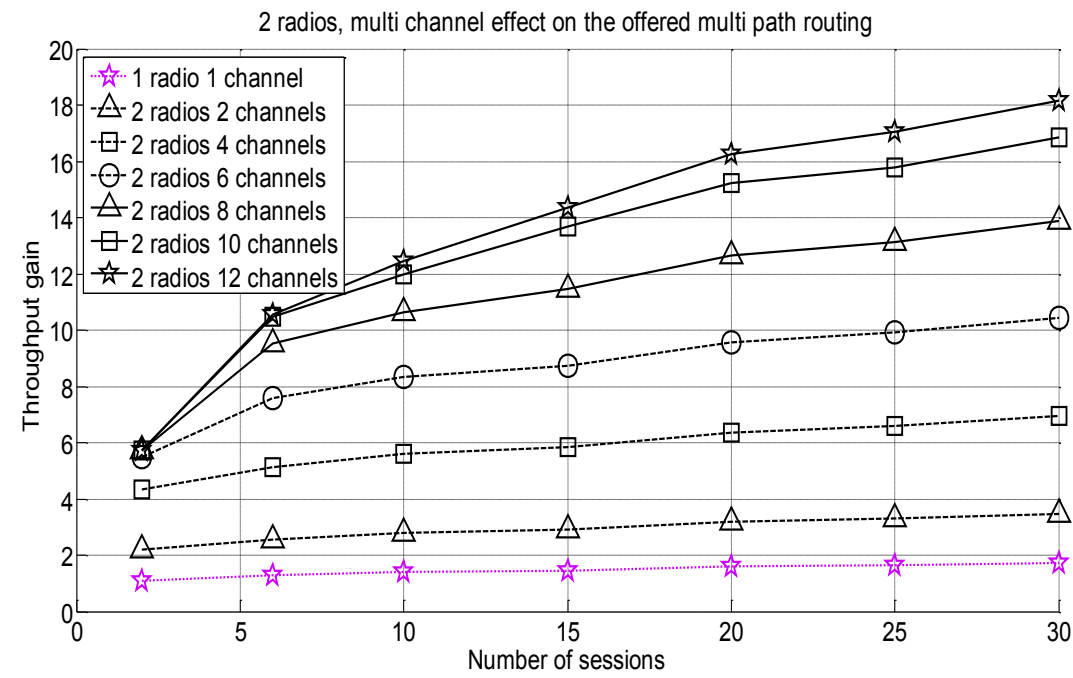

(b)

Figure 13. Effect of multi-channel on the throughput gain in conventional plus and random network when each node is equipped with 2 radios in 2 different routing scenarios: (a) Proposed single-path routing, (b) Proposed multi-path routing. 
Comparing Figure 12 (a) and (b), in a single-path routing the maximum throughput gain obtained is 4.5 and saturation capacity is 5 . While in a multipath routing these parameters are 8.2 and 6 respectively. Thus, in this case, multi-path routing approximately doubles the throughput gain capacity, but does not change the saturation capacity considerably.

The number of concurrent communications that each node can handle is equal to the number of radios the node has. Thus it is expected that increasing the number of radios would boost the throughput gain of the network. Figure 12 shows the throughput gain of the random network when two radios are deployed in each node. The comparison between Figure 12 and Figure 13 shows that in both cases of the single-path and the multi-path routing, utilization of the extra radio doubles the throughput gain capacity and saturation capacity at the same time. Finally, it implies that if there are $\mathrm{M}$ radios available in each node, the saturation capacity will be $4 \mathrm{M}$ in the single-path and $5 \mathrm{M}$ in the multi-path routing schemes.

\section{CONCLUSION}

We modeled the throughput gain of analog and conventional $\mathrm{NC}$ methods in a wireless ad-hoc network and evaluated the throughput gain of each $\mathrm{NC}$ scenario over the non-NC one. Our results show that conventional $\mathrm{NC}$ schemes which include cross topology $\mathrm{NC}$, could outperform traditional analog $\mathrm{NC}$ schemes. Thus, it is possible to prevent practical complexities of analog NC implementation, though obtaining its desired throughput gain at the same time. Then, we evaluated the effect of applying some routing methods in throughput gain of wireless ad-hoc networks and developed a routing method with a great potential to improve the throughput through the use of network coding. Our method is fast and could be effective in both single-path and multi-path scenarios.

\section{REFERENCES}

Ahlswede, R., Cai, N., Li, S. Y., \& Yeung, R. W. (2000). Network information flow. IEEE Transactions on Information Theory, 46(4), 1204-1216.

Argyriou, A. (2009). Wireless network coding with improved opportunistic listening. IEEE Transactions on Wireless Communications, 8(4), 20142023. 
Argyriou, A. (2010). Cross-layer and cooperative opportunistic network coding in wireless ad hoc networks. IEEE Transactions on Vehicular Technology, 59(2), 803-812.

Ayedi, M., Sellami, N., \& Siala, M. (2015, August). Throughput and energy optimization of a cooperative ARQ scheme using analog network coding. In Signal Processing Conference (EUSIPCO), 2015 23rd European (pp. 1466-1470). IEEE.

Chieochan, S., \& Hossain, E. (2013). Channel assignment for throughput optimization in multichannel multiradio wireless mesh networks using network coding. IEEE Transactions on Mobile Computing, 12(1), 118-135.

Choi, G., \& Yoon, W. (2016). Distributed joint rate control, resource allocation, and congestion control for throughput optimization in multirate multiradio multichannel wireless network with intersession/ intrasession network coding. Wireless Personal Communications, pp. 271-287.

Fragouli, C., Le Boudec, J. Y., \& Widmer, J. (2006). Network coding: An instant primer. ACM SIGCOMM Computer Communication Review, 36(1), 63-68.

Katti, S., Gollakota, S., \& Katabi, D. (2007). Embracing wireless interference: Analog network coding. ACM SIGCOMM Computer Communication Review, 37(4), 397-408.

Katti, S., Rahul, H., Hu, W., Katabi, D., Médard, M., \& Crowcroft, J. (2008). XORs in the air: Practical wireless network coding. IEEE/ACM Transactions on Networking (ToN), 16(3), 497-510.

Kim, T. H., Choi, H., \& Park, H. S. (2014, February). Centrality-based network coding node selection mechanism for improving network throughput. 16th International Conference on Advanced Communication Technology (pp. 864-867). IEEE.

Lee, U., Lee, S. H., Lee, K. W., \& Gerla, M. (2013). Understanding processing overheads of network coding-based content distribution in VANETs. IEEE Transactions on Parallel and Distributed Systems, 24(11), 23042318. 
Li, Z., \& Li, B. (2004, September). Network coding: The case of multiple unicast sessions. Allerton Conference on Communications, Vol. 16, p. 8.

Nazer, B., \& Gastpar, M. (2011). Reliable physical layer network coding. Proceedings of the IEEE, 99(3), 438-460.

Niati, R., Banihashemi, A. H., \& Kunz, T. (2012). Throughput and energy optimization in wireless networks: Joint MAC scheduling and network coding. IEEE Transactions on Vehicular Technology, 61(3), 1372-1382.

Shengli, Z., Liew, S. C., \& Lam, P. (2006). Hot topic: Physical-layer network coding. Proceedings of the 12th Annual International Conference on Mobile Computing and Networking. ACM.

Su, H., \& Zhang, X. (2009). Modeling throughput gain of network coding in multi-channel multi-radio wireless ad hoc networks. IEEE Journal on Selected Areas in Communications, 27(5), 593-605.

Teav, K. K., Zhou, Z., \& Vucetic, B. (2012, May). Throughput optimization for MIMO Y channels with physical network coding and adaptive modulation. Vehicular Technology Conference (VTC Spring), 2012 IEEE 75th (pp. 1-5). IEEE.

Wang, X., \& Garcia-Luna-Aceves, J. J. (2009). Embracing interference in ad hoc networks using joint routing and scheduling with multiple packet reception. Ad Hoc Networks, 7(2), 460-471.

Wu, Y., Chou, P. A., \& Kung, S. Y. (2005). Information exchange in wireless networks with network coding and physical-layer broadcast, Vol. 78. MSR-TR-2004.

Xie, L. F., Chong, P. H. J., \& Guan, Y. L. (2015). Performance analysis of network coding with virtual overhearing in wireless networks. IEEE Transactions on Vehicular Technology, 64(5), 2051-2061.

Yan, Q., Li, M., Yang, Z., Lou, W., \& Zhai, H. (2012). Throughput analysis of cooperative mobile content distribution in vehicular network using symbol level network coding. IEEE Journal on Selected Areas in Communications, 30(2), 484-492. 
Yang, H., Meng, W., Li, B., \& Wang, G. (2012, June). Physical layer implementation of network coding in two-way relay networks. 2012 IEEE International Conference on Communications (ICC) (pp. 671675). IEEE.

Zhang, X., \& Su, H. (2009). Network-coding-based scheduling and routing schemes for service-oriented wireless mesh networks. IEEE Wireless Communications, 16(4), 40-46. 


\section{APPENDIX}

\section{Optimization formula}

The complete set of optimization formula for LP1 is presented here.

\section{Maximize:}

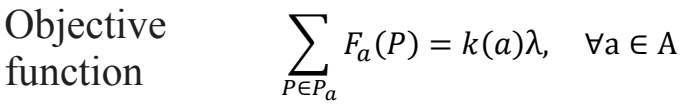

Fairness

constraint

$$
f(e)=\sum_{m \in M} f_{m}^{U}(e)+\sum_{m \in M} \sum_{L \in L(e)} f_{m}^{N C}(L), \quad \forall e \in E
$$

Link flow

constraint

$$
f(e)=\sum_{a \in A} \sum_{P \in P_{a}: P \ni e} F_{a}(P), \quad \forall e \in E
$$

Flow conservation

$$
\sum_{e \in E^{+}(v)} f(e)-\sum_{e \in E^{-}(v)} f(e)
$$

constraint

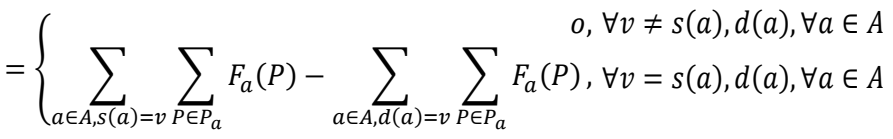

Transmission interference constraint

$$
x_{m}^{U}(e)=\left\{\begin{array}{rr}
\frac{f_{m}^{U}(e)}{c_{m}(e)}, & c_{m}(e)>0 \\
0, & \text { otherwise }
\end{array}\right.
$$

$$
\begin{aligned}
& x_{m}^{N C}(L)=\left\{\begin{aligned}
\frac{f_{m}^{N C}(L)}{\min _{e \in L}\left\{c_{m}(e)\right\}}, & \min _{e \in L}\left\{c_{m}(e)\right\}>0 \\
0, & \text { otherwise }
\end{aligned}\right. \\
& \sum_{e^{\prime} \in E^{C}(e)} x_{m}^{U}\left(e^{\prime}\right)+\sum_{L \in L_{m}^{C}(e)} x_{m}^{N C}(L) \leq 1, \quad \forall m \in M \& \forall e \in E \cup I
\end{aligned}
$$

Link capacity $\quad 0 \leq f_{m}^{N C}(L) \leq \min _{e \in L}\left\{c_{m}(e)\right\}, \quad \forall m \in M \& \forall \mathrm{L} \in \boldsymbol{L}$ constraint

$$
0 \leq f_{m}^{U}(e) \leq c_{m}(e), \quad \forall m \in M \& \forall e \in E
$$

Node radio constraint

$$
\sum_{m \in M}\left(\sum_{e \in E(v)} x_{m}^{U}(e)+\sum_{\mathrm{L} \in \cup_{e \in E(v) L} L(e)} x_{m}^{N C}(L)\right) \leq W(v)
$$

